\title{
Detecting Wedge Shaped Defects in Polarimetric Images of the Retinal Nerve Fiber Layer
}

\author{
Koen Vermeer ${ }^{1}$, Frans $\operatorname{Vos}^{1,3}$, Hans Lemij ${ }^{2}$, and Albert Vossepoel ${ }^{1}$ \\ 1 Pattern Recognition Group, Delft University of Technology \\ Lorentweg 1, 2628 JC Delft, The Netherlands \\ \{koen, frans, albert\}@ph.tn.tudelft.nl \\ 2 Rotterdam Eye Hospital, Schiedamsevest 180, 3011 BH Rotterdam, The Netherlands \\ lemij@wxs.nl \\ 3 Academic Medical Center, Department of Radiology \\ P.O. Box 22660, 1100 DD Amsterdam, The Netherlands
}

\begin{abstract}
Wedge shaped defects of the retinal nerve fiber layer (RNFL) may occur in glaucoma. Currently, automatic detection of wedge shaped defects in Scanning Laser Polarimetry images of the retinal nerve fiber layer is unavailable; an automatic classification is currently based only on global parameters, thereby ignoring important local information. Our method works by a modified dynamic programming technique that searches for locally strong edges with a preference for straight edges. These edges are initially classified based on their strength and then combined into wedge shaped defects. The results of our method on a limited set of 45 images yields a sensitivity of $88 \%$ and a specificity of $92 \%$. More importantly, it shows that it is possible to automatically extract local RNFL defects such as wedges.
\end{abstract}

\section{Introduction}

Glaucoma is a fairly common eye disease that leads to thinning and loss of the retinal nerve fiber layer ((R)NFL). As a result, visual field defects develop. If untreated, the disease will lead to blindness. Many methods are available for diagnosis and screening of glaucoma, including Scanning Laser Polarimetry (SLP). In this method the thickness of the NFL is calculated by measuring the retardation of reflecting polarized light [1].

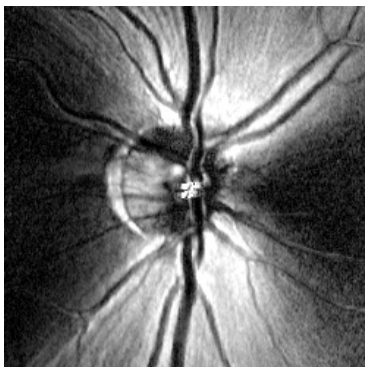

(a)

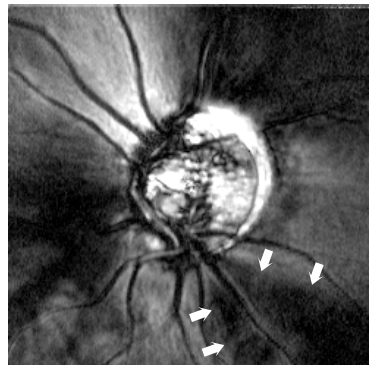

(b)

Fig. 1. Retardation images. (a) Healthy eye. (b) Wedge shaped defect, marked by white arrows. 


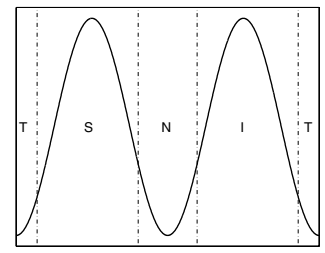

(a)

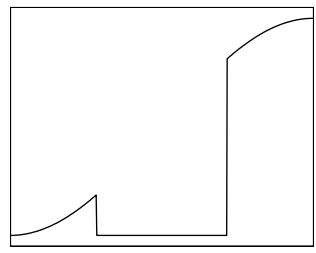

(b)

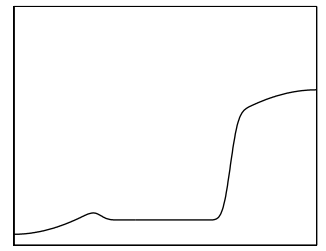

(c)

Fig. 2. (a) TSNIT-graph. (b) Ideal wedge. (c) Less ideal wedge.

A commercially available SLP based device is the GDX 1 , which was used to acquire the images in this paper. Our version was a prototype that included a so-called variable cornea compensator. An example of a retardation image is shown in Fig. 1(a). All images in this paper are stretched to enhance the contrast. The circular object in the center of the image is the optic disc, where the NFL exits the eye.

Currently, parameters based on global properties of the retardation image are used for computer aided diagnosis. However, there is more information to the trained observer. An important local abnormality that is not detected by current computer algorithms is the wedge shaped defect. An example is given in Fig.[1(b)](located at 5 o'clock, marked by white arrows). A wedge shaped defect, which can precede visual field loss, is caused by localized loss of grouped nerve fibers. Wedges almost always signify an abnormality, although this may not always be glaucoma [2].

This paper aims to present a new detection scheme for wedge shaped defects, which can help in the diagnosis of glaucoma. To the best of our knowledge, no such automated wedge detection currently exists. Our method uses a polar representation of the retardation image and novel dynamic programming techniques. It opens a new way to interpret the information contained in these images.

\section{Method}

\subsection{Model}

A TSNIT-graph is a graph of the thickness of the RNFL at a certain distance from the optic disc at all angles, starting at the Temporal area, running through the Superior, Nasal, Inferior and again the Temporal area. The TSNIT-graph ideally looks like the double sinus of Fig. 2(a), Only the superior part of the temporal half will be displayed in the next figures, because clinically significant wedge defects mostly occur on the temporal side. Also, for the sake of argument, the superior and inferior sides can be considered similar. The ideal wedge shaped defect is modelled in Fig. 2(b), It is characterized by two opposing strong step edges.

The NFL of real eyes, however, is less ideal than this. The general NFL thickness can be much less, resulting in less contrast. Also, the detected NFL thickness inside a wedge can be significantly larger than zero and the step edges may be blurred. Figure 2(c) shows all these deviations. Therefore, when looking for the edges of a wedge, one should look for steep slopes.

\footnotetext{
${ }^{1}$ GDx Nerve Fiber Analyzer, Laser Diagnostic Technologies, Inc., San Diego, CA,
} http://www.laserdiagnostic.com 
The wedges are modelled by two radially orientated, opposing edges. These edges start at the outside of the image, running approximately straight to the border of the optic disc. The edges can be observed most clearly at the outside of the image, because on these locations, they are less frequently occluded by blood vessels. By definition, wedge defects must extend to the border of the optic disc. Moreover, one of the edges will have a steeper slope and will thus be stronger than the other; the former one will be called 'strong', the latter 'weak'.

\subsection{Outline}

We will locate the wedges by applying the next scheme:

- Preprocessing.

- Edge detection.

- Matching edges.

The preprocessing step consists of the detection and removal of blood vessels and a transformation of the image coordinates. In the edge detection step, the locally strong edges are located. In the last step, the edges that have been found are classified based on their strength and corresponding edges are matched. In the following sections, each step is described thoroughly.

\subsection{Preprocessing}

Blood vessels show the same strong edges as do wedges. In addition, the NFL is occluded by them. Therefore, blood vessel detection is required. In Fig. 3(a), the result of our blood vessel detection scheme is shown. A comprehensive description is given in [3]. We estimate the NFL in areas occluded by the blood vessels by a Gaussian extrapolation of the surrounding neighborhood (see Fig. 3(b)).

Since the edges of the wedge are orientated radially, we introduce a polar representation of the NFL. The diameter of the optic disc in the center of the image, which is of no interest to us, is generally at least one quarter of the image. Therefore, a circle with a radius of one quarter of the image size is ignored. The coordinate transformation results in Fig. 4. The far left side of the polar representation corresponds to the 6 o'clock

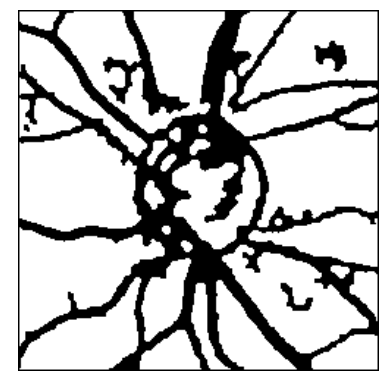

(a)

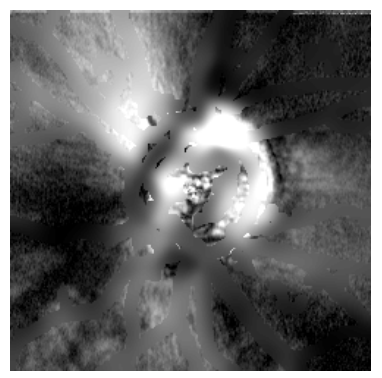

(b)

Fig. 3. Estimating the NFL. (a) Result of blood vessel detection. (b) Estimation of NFL at blood vessel areas. 


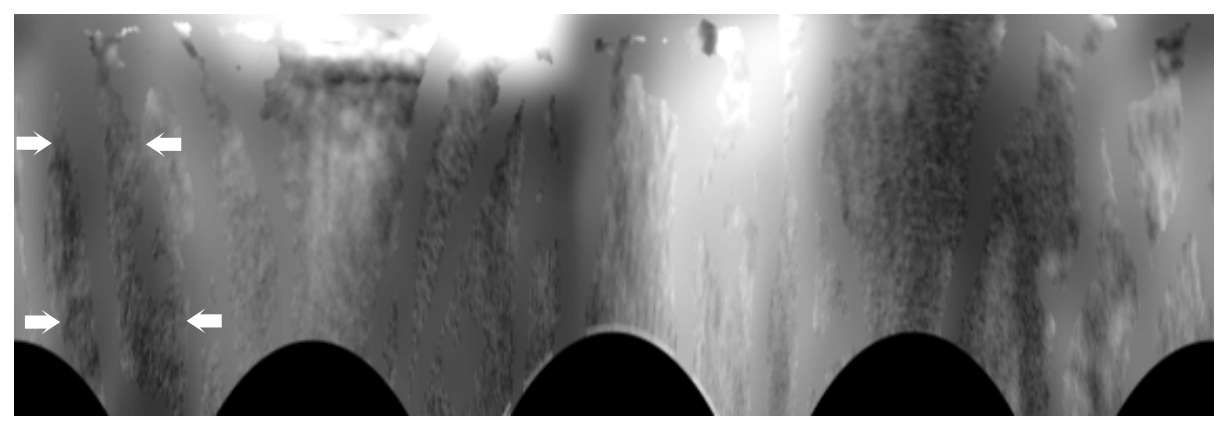

Fig. 4. Polar representation. The wedge shaped defect is marked by white arrows.

position in the original image. The positive $\mathrm{x}$-direction of the polar image corresponds to an anti-clockwise rotation. In this polar image, the wedge is located on the left side of the image (marked by white arrows). Since wedges almost exclusively occur on the temporal half ( $97 \%$ according to [4]), we will focus only on the left half of the polar image.

\subsection{Finding Edges}

The procedure to identify edges consists of three steps:

1. Texture removal.

2. Differentiation.

3. Edge detection.

The image consists of both larger scale 'objects' such as the local thickness of the NFL and smaller ones that are superimposed (texture). To remove the ripple (texture) without removing the larger (objects) edges, the average of the closing and the opening operator (see [5]) is taken. Wedges, by definition, are larger than the blood vessels. Also, there is a need to retain spatial coherence in the vertical direction. Consequently, an ellipse-shaped structuring element of size 12x3 (hor. x vert.) pixels is used, resulting in Fig. 5(a).

Differentiating is done horizontally by calculating the central difference (see Fig. 5(b)].

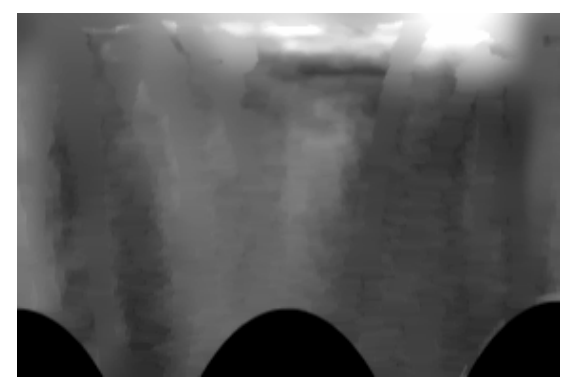

(a)

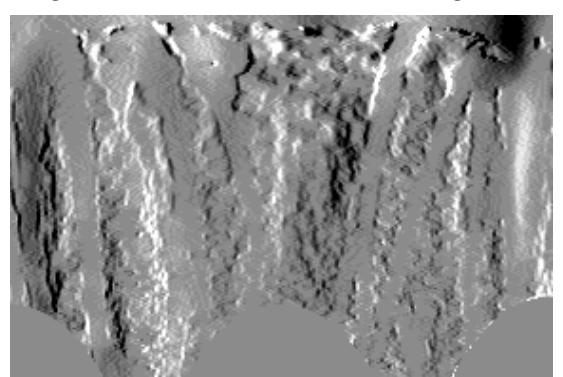

(b)

Fig. 5. (a) Image with texture removed. (b) Central difference in $x$ direction. The white pixels have a large positive difference while the black pixels have a large negative difference. 


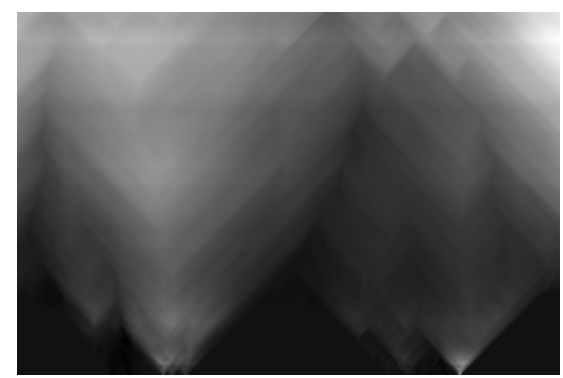

(a)

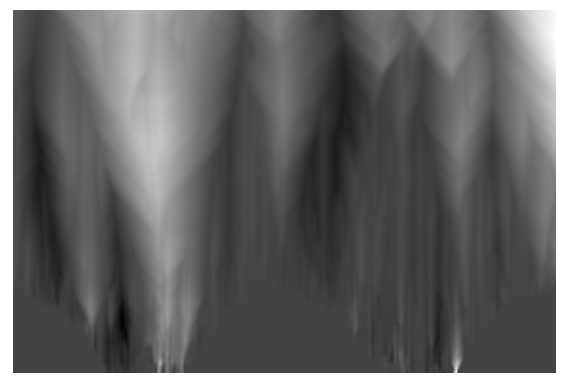

(b)

Fig. 7. Cumulative cost matrices. Each horizontal line is normalized. (a) Without penalty. (b) With penalty.

For our images, the bottom of the image is of most interest. This corresponds to the outside of the normal images, where less occlusion occurs and the edges of the wedge are better visible. We therefore calculate the cost function starting at the bottom, working our way up, and then select our paths starting at the top. The cumulative cost function used is similar to that of the example:

$$
\phi_{n+1}^{i}=c_{n+1}^{i}+\max \left(\phi_{n}^{i-1}-\operatorname{pen}, \phi_{n}^{i}, \phi_{n}^{i+1}-\text { pen }\right),
$$

where pen is the penalty on a diagonal connection. If we omit the penalty, and apply the cost function to the example image, Fig.7(a) results (for display purposes only, each horizontal line is normalized by dividing all pixels on a line by the maximum pixel value on that line). Introducing a penalty of, for example, 10 (about twice the largest pixel value in the differentiated image) gives better results, as can be seen in Fig. 7(b)] High values are propagated less in horizontal direction, thereby favoring straight paths.

At last, we now calculate the route and the endpoint for each pixel in the top row and keep the best route for each endpoint, defining the detected edges. The same can be done for the edges in the opposing direction by negating our differentiated image and following the same procedure. The result is displayed in Fig. 8(a)

The strength $s(i)$ of a pixel on an edge, at distance $i$ from the bottom, can be defined in two ways: Absolute (the value of the pixel in the differentiated image) or relative (the absolute difference divided by the maximum of the left and right pixel value in the original polar image). The average strength of a part of an edge with length $l$, starting

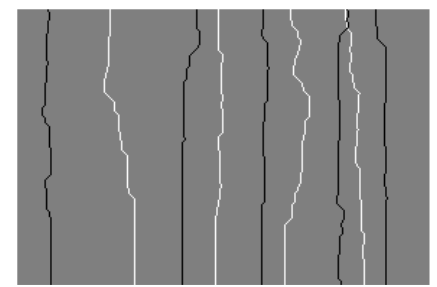

(a)

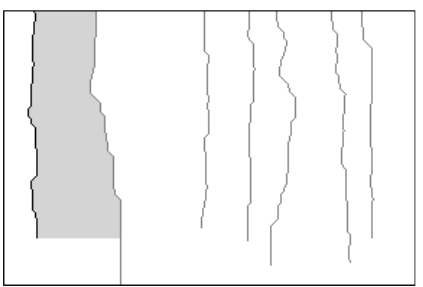

(b)

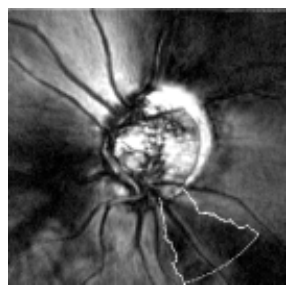

(c)

Fig. 8. (a) Located edges. White edges have a positive difference, black edges have a negative difference. (b) Matched edges (grey area). The black line (left from the grey area) is a strong edge, the other (grey) lines are weak edges. (c) Detected wedge shown overlayed on retardation image. 
from the bottom is defined by $s_{\operatorname{avg}}(l)=\frac{1}{l} \int_{0}^{l} s(i) \mathrm{d} i$. We now define the strength of the edge as $\max \left(s_{\operatorname{avg}}(l)\right)$ for $\frac{2}{3} L \leq l \leq L$, with $L$ being the total length of the edge. The minimum length of $\frac{2}{3} L$ allows for optic disc diameters of up to half the image size.

By putting thresholds $(\theta)$ on the strength of the edge, we define two edges types: Strong edges (strength $\geq \theta_{s}$ ) and weak edges (strength $\geq \theta_{w}$ ), with $\theta_{s}>\theta_{w}$. Edges with a strength smaller than $\theta_{w}$ are ignored.

\subsection{Matching Edges}

For the image at hand, we now have both strong and weak edges, in both directions. Matching edges is done by starting at a strong edge and looking for an opposing weak edge that is located at the correct side of the strong edge within a distance of $60^{\circ}([4])$.

The result for the example image is shown in Fig. 8(b). When drawn in the original retardation image, Fig. 8(c) results.

\section{Results}

The images recorded with the GDx are $256 \times 256$ pixels at a quantization of 8 bits per pixel. The viewing angle is $15^{\circ}$; the sampling density is approximately 59 pixels $/ \mathrm{mm}$.

To show the usefulness of our detection scheme, we used a total of 45 images of both normal and glaucomatous eyes. Eight of these 45 images showed one or more wedges shaped defects, defined by a physician. Analysis of the edges of these wedges resulted in an relative threshold for the strong edges of 0.14 . For the weak edges, both a relative threshold of 0.033 and an absolute threshold of 1.5 were found, which had to be satisfied both. The results of the detection scheme, presented in Table 1 are on a per image basis, meaning that if an eye had a wedge and the algorithm detected one, it was counted as a true positive. Consequently, eyes with more than one wedge were counted as one. The approach was chosen with a diagnosis setup in mind, where the eventual conclusion concerns the whole eye.

Of the 8 images with wedge shaped defects, 7 were detected. So, the sensitivity was $88 \%$, but because of the small number of images with wedges in our set, the $95 \%$ confidence interval of the sensitivity was quite large (47\%-99\%). Three false positives resulted, giving a specificity of $92 \%$, with a smaller $95 \%$ confidence interval of $77 \%$ $98 \%$. By changing the parameters, such as the thresholds, these values can be adjusted.

Table 1. Results. (a) True positive, false positive, false negative and true negative results. (b) Sensitivity and specificity, including their estimated $95 \%$ confidence interval.

\begin{tabular}{|l|l|l|l|}
\hline & $\begin{array}{l}\text { Wedge } \\
\text { visible }\end{array}$ & $\begin{array}{l}\text { Wedge } \\
\text { not visible }\end{array}$ & Totals \\
\hline Wedge detected & 7 & 3 & 10 \\
\hline Wedge not detected & 1 & 34 & 35 \\
\hline Totals & 8 & 37 & 45 \\
\hline
\end{tabular}

(a)

\begin{tabular}{|l|l|l|}
\hline & Estimated & $\begin{array}{l}95 \% \text { confidence } \\
\text { interval }\end{array}$ \\
\hline Sensitivity & 0.88 & $0.47-0.99$ \\
\hline Specificity & 0.92 & $0.77-0.98$ \\
\hline
\end{tabular}

(b) 


\section{Conclusion}

By modelling wedge shaped defects and using a novel dynamic programming technique, it proved to be possible to automatically detect wedge shaped defects. While the detection scheme needs more testing, the preliminary results are promising. Our method combines good sensitivity with great specificity. It also shows that it is possible to extract more information from the retardation images in an automated way than is currently done. This can greatly help computer assisted diagnosis.

\subsection{Discussion}

The visibility of wedges in advanced glaucomatous eyes can be much worse than in eyes in which the NFL is still (largely) unaffected [4]. Current methods can detect glaucomatous eyes quite well, but perform worse in cases of early glaucoma. Therefore, application of this automated wedge detection will be most beneficial in cases where conventional methods do not classify an eye as glaucomatous.

With a prevalence of glaucoma of approximately $2 \%$ in the general Caucasian population, screening for the disease requires a high specificity, at the expense of sensitivity, to avoid an unacceptably high overcalling by any diagnostic tool. By adjusting the threshold values, our algorithm can be tuned to the specific needs of the setting in which it is used.

\section{References}

1. Andreas W. Dreher and Klaus Reiter. Scanning laser polarimetry of the retinal nerve fiber layer. In Polarization Analysis and Measurement, volume 1746 of Proc. SPIE, pages 34-41, 1992.

2. Jost B. Jonas and Albert Dichtl. Evaluation of the retinal nerve fiber layer. Surv Ophthalmology, 40(5):369-378, Mar-Apr 1996.

3. K.A. Vermeer, F.M. Vos, H.G. Lemij, and A.M. Vossepoel. A model based method for retinal blood vessel detection. Internal report.

4. Jost B. Jonas and Dennis Schiro. Localised wedge shaped defects of the retinal nerve fibre layer in glaucoma. Br J Ophthalmology, 78(4):285-290, Apr 1994.

5. P.W. Verbeek, H.A. Vrooman, and L.J. van Vliet. Low-level image processing by min-max filters. Signal Processing, 15(3):249-258, 1988.

6. J. J. Gerbrands. Segmentation of Noisy Images. PhD thesis, Delft University of Technology, 1988.

7. Davi Geiger, Alok Gupta, Luiz A. Costa, and John Vlontzos. Dynamic programming for detecting, tracking and matching deformable contours. IEEE Transactions on Pattern Analysis and Machine Intelligence, 17(3):294-302, Mar 1995.

8. Dimitri P. Bertsekas. Dynamic Programming. Prentice-Hall, Inc., 1987. 